Research Article

\title{
Dynamic Response Analysis of a Forced Fractional Viscoelastic Beam*
}

\author{
Kenan Yildirim (iD ${ }^{1}$ and Sertan Alkan (iD) ${ }^{2}$ \\ ${ }^{1}$ Mus Alparslan University, Merkez, Mus, Turkey \\ ${ }^{2}$ Iskenderun Technical University, İskenderun, Hatay, Turkey \\ Correspondence should be addressed to Kenan Yildirim; k.yildirim@alparslan.edu.tr
}

Received 3 September 2021; Revised 12 November 2021; Accepted 4 December 2021; Published 15 December 2021

Academic Editor: Fairouz Tchier

Copyright (C) 2021 Kenan Yildirim and Sertan Alkan. This is an open access article distributed under the Creative Commons Attribution License, which permits unrestricted use, distribution, and reproduction in any medium, provided the original work is properly cited.

\begin{abstract}
In this paper, dynamic response analysis of a forced fractional viscoelastic beam under moving external load is studied. The beauty of this study is that the effect of values of fractional order, the effect of internal damping, and the effect of intensity value of the moving force load on the dynamic response of the beam are analyzed. Constitutive equations for fractional order viscoelastic beam are constructed in the manner of Euler-Bernoulli beam theory. Solution of the fractional beam system is obtained by using Bernoulli collocation method. Obtained results are presented in the tables and graphical forms for two different beam systems, which are polybutadiene beam and butyl B252 beam.
\end{abstract}

\section{Introduction}

Theory and applications of beams are very important research area due to its wide usage areas in applied sciences. Especially after starting the space adventure of the mankind, the demand to more resistant structures has great importance. Beams are generally modeled based on Euler-Bernoulli beam theory, which is called classical beam theory. The background of beam theory goes on Newton's second law and some different aspects of beams, such as modeling, analysis of bending-buckling, and reinforcement and control, are hot topics of research papers since the beginning of the nineteenth century. The books can provide a general overview about the Euler-Bernoulli beam theory, please see [1-3]. Some important studies related to beams modeled in the sense of classical beam theory are also summarized as follows, but not limited to [4-15]. The beam systems in [1-15] have the integer order derivatives of the state function. In the beginning of 1930s, fractional derivative was introduced for describing the constitutive relation of some beam materials [16], and after 1980s, since fractional order equations have good memory and can be used to describe material properties more accurately with fewer parameters, they are considered to be good mathematical models for describing the dynamic mechanical behavior of materials [17]. In [18], the dynamic behavior of the thin plates resting on a fractionally damped viscoelastic foundation subjected to a moving point load is investigated and results show that the damping of the foundation system increases with increasing the order of the fractional derivative, which leads to a decrease in the dynamic response. In [19], the dynamic response spectra of fractionally damped viscoelastic beams subjected to concentrated moving load are presented and results reveal that with an increase in the order of the fractional derivative, the system damping of the system increases and the dynamic amplification factor (DAF) decreases, especially in the dynamic zone of the sweep parameter. In [20], the precise integration method (PIM) is extended to numerically integrate the equation of motion with fractional terms, which offers high accuracy and obtained numerical results indicate the viscoelastic dampers can enhance the seismic performance of structures significantly. In [21], the nonstationary free vibration and nonlinear dynamic behavior of the viscoelastic nanoplates are analyzed. Obtained results show that the viscoelastic modelbased vibration is nonstationary unlike the elastic model. 
Moreover, the damping mechanism of the viscoelasticity is amplitude dependent and the contribution of the viscoelastic damping terms at higher forcing conditions becomes noticeable. On the other hand, several numerical methods are developed and employed for better analyzing the fractional mechanical systems. Widely used methods for fractional systems are finite element method [22], Galerkin method [23], variational iteration method [24], and multiscale method $[25,26]$. Especially, papers existing in the literature, which include a solution method for analyzing the dynamic response of a fractional order beam system, can be shortly listed as [19, 27-30]. In [19], the authors combined Galerkin method and Newton-Raphson method for analyzing the vibration of a fractional beam equation and they compared the results for only seeing the effects of fractional or integer derivatives. In [27], the author considered the dynamic response analyzing of a fractional order viscoelastic beam by means of green function method. In [27], the author only compared the results based on changes on the fractional derivative between $(0,1)$. In [28], the authors employed the Adomian decomposition method for solving a fractional beam equation and they only observed the effect of the order of fractional derivative. In [29], the authors used the dynamic green function method for analyzing the dynamic response in a fractional beam equation and the beam equation does not include the damping term. Results are simulated for only indicating the effects of order of fractional derivative. In [30], the author employed the green function method for a fractional viscoelastic beam system subjected to a base excitation. After obtaining the solution, the author compared the results corresponding to different fractional order derivative. By comparing the present study with the studies existing in the literature, objectives of the present study are expressed as follows:

(i) In this paper, Bernoulli collocation method is firstly employed for analyzing the fractional viscoelastic beam equation. In the literature, especially for the fractional beam systems, green function method, Galerkin method, Newton-Raphson method, Adomian decomposition method, and Bernoulli collocation method in this paper were used, but by comparing these five methods, it is clear that Bernoulli collocation method is new and has less computational process and less work.

(ii) In the literature, the authors only considered and discussed the effects of order of fractional derivative on the dynamic response. But, we discussed both the effects of the order of fractional order derivative and the effects of damping coefficient term and the effect of density of moving force load. So, it is said that the present study has wider perspective than other studies.

(iii) Also, in the literature, results are obtained for one beam system. In this paper, effects of order of fractional derivative, effects of damping coefficient term, and the effect of density of moving force load are observed and compared for two different beam systems which are polybutadiene beam and butyl B252 beam.

For theoretical and experimental review about the fractional Euler-Bernoulli beams, please see [31]. Specifically, in the present paper, displacement analysis of a forced fractional viscoelastic beam is studied. External moving force load perfectly moves on the beam with the velocity $v(t)$ from the left edge to the right edge of the beam. The solution of the fractional beam system is obtained by means of Bernoulli collocation method. The main advantage of the Bernoulli collocation method is that employing the Bernoulli polynomials is easier than Chebyshev, Bessel polynomials, and Haar wavelets [32-34]. These advantages of Bernoulli polynomials provide us for obtaining the solution by making less computational process in shorter time. In the step of employing the Bernoulli collocation method, some external moving force loads having different load intensities are considered and also the effects of internal damping and fractional order of the derivative are searched for a fractional beam system. In the simulations, two different beam systems, which are polybutadiene beam and butyl B252 beam, are taken into account for being compared each other in the aspects of internal damping effects and resistance to effect of external moving force. Comparison results of the beam systems are presented in tables and graphics. The rest of the paper is organized as follows: in the next section, definition of the displacement analysis problem for a fractional viscoelastic beam is presented and scheme of the beam is overviewed. In the third section, short definition of the fractional derivative in the Caputo sense is introduced. In the fourth section, Bernoulli collocation method is explained and adopted to the present problem. In the fifth section, obtained results are given and discussions are made in the light of employing the Bernoulli collocation method to fractional viscoelastic beam system.

\section{Definition of the Problem}

The motion equation of the fractional viscoelastic homogeneous beam is obtained by considering the Euler-Bernoulli beam theory by ignoring shear deformation factor and rotary inertia of the beam. The beam is considered as a uniform viscoelastic beam and mechanical energy dissipation inside the beam is modeled by fractional order differential equations. By taking into account the [35], stressstrain constitutive relation of a fractional viscoelastic beam is given as follows:

$$
\sigma=E \varepsilon(t)+E_{\gamma}^{\prime} D_{t}^{\gamma}[\varepsilon(t)]=E\left(\varepsilon+\mu_{\gamma} \frac{d^{\gamma} \varepsilon(t)}{\mathrm{d} t^{\gamma}}\right),
$$

in which $E$ is the Young's modulus of the viscoelastic beam, $\mu_{\gamma}$ is the damping coefficient, and $D_{t}^{\gamma}$ is the fractional derivative operator with the order $\gamma$ with respect to $t$. The simply supported viscoelastic beam initially is at rest and nondeformed. The beam is subjected to a horizontally moving constant force load with the velocity $v(t)$ from the left edge to right edge of the beam, respect to $x$ axis. In the 
light of [27], let us introduce the formulation of a fractional viscoelastic beam structure illustrated in Figure 1 .

$$
A \rho \frac{\partial^{2} w(t, x)}{\partial t^{2}}+\operatorname{EI} \mu_{\gamma}\left[\frac{d^{\gamma}}{d t^{\gamma}} \frac{\partial^{4} w(t, x)}{\partial x^{4}}\right]+\mathrm{EI} \frac{\partial^{4} w(t, x)}{\partial x^{4}}=P \delta(x-v(t))
$$

in which $w$ is the deflection of the viscoelastic beam in $\mathscr{C}=\{(t, x): t \in(0, \mathrm{tf}), x \in(0, \ell)\}, t$ is the time variable, $t_{f}$ is the final time observed duration, $x$ is the space variable, $\ell$ is the length of the viscoelastic beam, $A$ is the cross-section area of the structure, $\rho$ is the material mass density of the viscoelastic beam, $I$ is the axial moment of inertia of the beam, $P$ is a constant showing intensity of the external moving force load, $\delta$ is the Dirac-delta function, and $v(t)$ is the velocity of the moving force load with the condition $0 \leq v(t) \leq \ell$. Equation (2) is subjected to the following boundary conditions:

$$
w(t, x)=0, w_{x x}(t, x)=0 \text { at } x=0, \ell,
$$

and the following initial conditions:

$$
w(t, x)=w_{0}(x), w_{t}(t, x)=w_{1}(x) \text { at } t=0,
$$

in which $w_{0}(x) \in H^{1}(0, \ell)=\left\{w_{0}(x) \in L^{2}(0, \ell): \partial w_{0}(x) /\right.$ $\left.\partial x \in L^{2}(0, \ell)\right\}, w_{1}(x) \in L^{2}(0, \ell) . L^{2}(\mathscr{C})$ means to squareintegrable functions space in the manner of Hilbert in the domain $\mathscr{C}$ in the Lebesgue sense with the following norm and inner product:

$$
\|\eta\|^{2}=\langle\eta, \eta\rangle, \quad\langle\eta, \rho\rangle_{\mathscr{C}}=\int_{\mathscr{C}} \rho \eta d \mathscr{C} .
$$

Let us assume that

$$
w(t, x)=\sum_{n=1}^{N} z_{n}(t) \sqrt{2} \sin \left(\frac{n \pi x}{\ell}\right) .
$$

After substituting the equations (6) into (2) and multiplying both sides of equation (2) with $\sqrt{2} \sin (n \pi x / \ell)$, integrating on $(0, \ell)$, we obtain the following ordinary differential equation as follows:

$$
A \rho z_{n}^{\prime \prime}(t)+\operatorname{EI}(n \pi)^{4} \mu_{\gamma}\left[\frac{d^{\gamma}}{\mathrm{d} t^{\gamma}} z_{n}(t)\right]+\operatorname{EI}(n \pi)^{4} z_{n}(t)=P \sqrt{2} \sin \left(\frac{n \pi v(t)}{\ell}\right), \quad n=1, \ldots, N
$$

Equation (7) is subjected to the following initial conditions:

$$
z_{n}(0)=\sqrt{2} \int_{0}^{\ell} w_{0}(x) \sin \left(\frac{n \pi x}{\ell}\right) \mathrm{d} x, \quad z_{n}^{\prime}(0)=\sqrt{2} \int_{0}^{\ell} w_{1}(x) \sin \left(\frac{n \pi x}{\ell}\right) \mathrm{d} x .
$$

\section{The Fractional Derivative in the Caputo Sense}

Definition. The Caputo definition of the fractional-order derivative is

$$
D^{\gamma} f(x)=\frac{1}{\Gamma(n-\gamma)} \int_{0}^{x} \frac{f^{(n)}(t)}{(x-t)^{\gamma+1-n}} \mathrm{~d} t, \quad n-1<\gamma \leq n, n \in \mathbb{N}
$$

where $\gamma>0$ is the order of the derivative and $n$ is the smallest integer greater than $\gamma$. For the Caputo derivative, we have 


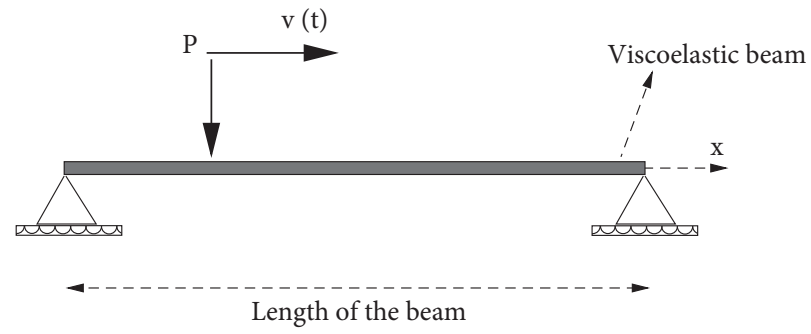

FIgURE 1: Schematic of the viscoelastic beam under moving force load $P$ with the velocity $v(t)$.

$D^{\gamma} C=0, \quad C$ is constant,

$D^{\gamma} x^{q}= \begin{cases}0, & \text { for } q \in \mathbb{N}_{0} \text { and } q<\lceil\gamma\rceil, \\ \frac{\Gamma(q+1)}{\Gamma(q+1-\gamma)} x^{q-\gamma}, & \text { for } q \in \mathbb{N}_{0} \text { and } q \geq\lceil\gamma\rceil \text { or } q \notin \mathbb{N} \text { and } q>\lfloor\gamma\rfloor .\end{cases}$

\section{Bernoulli Collocation Method}

The recurrence relation of the Bernoulli polynomials is defined by

$$
B_{n}(x)=2 x B_{n-1}(x)+B_{n-2}(x) .
$$

For $n \geq 3 ., B_{1}(x)=1, B_{2}(x)=2 x$. The first few Bernoulli polynomials are

$$
\begin{aligned}
& B_{1}(x)=1, \\
& B_{2}(x)=x-\frac{1}{2}, \\
& B_{3}(x)=x^{2}-x-\frac{1}{6}, \\
& B_{4}(x)=x^{3}-\frac{3}{2} x^{2}+\frac{x}{2}
\end{aligned}
$$

Our goal is to get the approximate solution as the truncated Bernoulli series defined by

$$
y(x)=\sum_{n=1}^{N+1} c_{n} B_{n}(x),
$$

where $B_{n}(x)$ denotes the Bernoulli polynomials; $c_{n}(1 \leq n \leq N+1)$ are the unknown coefficients for Bernoulli polynomial, and $N$ is any positive integer which possess $N \geq m$. Let us assume that linear combination of Bernoulli polynomials equation (14) is an approximate solution of equation (7). Our purpose is to determine the matrix forms of equation (7) by using (14). Firstly, we can write Bernoulli polynomials (12) in the matrix form

$$
\mathbf{B}(x)=\mathbf{T}(x) \mathbf{M},
$$

where $B(x)=\left[B_{1}(x) B_{2}(x) \cdots B_{N+1}(x)\right], \quad \mathbf{T}(x)=\left(1 x x^{2}\right.$ $\left.x^{3} \ldots x^{N}\right), \mathbf{C}=\left(c_{1} c_{2} \cdots c_{N+1}\right)^{T}$, and

$$
\mathbf{M}=\left(\begin{array}{ccccccccc}
1 & -\frac{1}{2} & \frac{1}{6} & 0 & -\frac{1}{30} & 0 & \frac{1}{42} & 0 & -\frac{1}{30} \\
0 & 1 & -1 & \frac{1}{2} & 0 & -\frac{1}{6} & 0 & \frac{1}{6} & 0 \\
0 & 0 & 1 & -\frac{3}{2} & 1 & 0 & -\frac{1}{2} & 0 & \frac{2}{3} \\
0 & 0 & 0 & 1 & -2 & \frac{5}{3} & 0 & -\frac{7}{6} & 0 \\
0 & 0 & 0 & 0 & 1 & -\frac{5}{2} & \frac{5}{2} & 0 & -\frac{7}{3} \\
0 & 0 & 0 & 0 & 0 & 1 & -3 & \frac{7}{2} & 0 \\
0 & 0 & 0 & 0 & 0 & 0 & 0 & 0 & 1
\end{array}\right) .
$$

The matrix form of equation (14) by a truncated Bernoulli series is given by

$$
y(x)=\mathbf{B}(x) \mathbf{C} .
$$

By using equations (15) and (17), the matrix relation is expressed as

$$
\begin{aligned}
y(x) & \cong y_{N}(x)=\mathbf{T}(x) \mathbf{M C} \\
y^{(\gamma)}(x) & \cong y_{N}^{(\gamma)}(x)=\mathbf{T}(x) \mathbf{X}_{(\gamma)}(x) \mathbf{D}_{(\gamma)} \mathrm{MC}, \\
y^{\prime \prime}(x) & \cong y_{N}^{\prime \prime}(x)=\mathbf{T}(x) \mathbf{D}^{2} \mathbf{M C}
\end{aligned}
$$


where

$$
\begin{aligned}
& \mathbf{X}_{(\gamma)}(x)=\left[0, x^{1-\gamma}, x^{2-\gamma}, \ldots, x^{N-\gamma}\right] \\
& \mathbf{D}=\left[\begin{array}{cccccccc}
0 & 1 & 0 & 0 & 0 & 0 & \cdots & 0 \\
0 & 0 & 2 & 0 & 0 & 0 & \cdots & 0 \\
0 & 0 & 0 & 3 & 0 & 0 & \cdots & 0 \\
0 & 0 & 0 & 0 & 4 & 0 & \cdots & 0 \\
0 & 0 & 0 & 0 & 0 & 5 & \cdots & 0 \\
0 & 0 & 0 & 0 & 0 & 0 & \cdots & 0 \\
\vdots & \vdots & \vdots & \vdots & \vdots & \ddots & \ddots & N \\
0 & 0 & 0 & 0 & 0 & 0 & \cdots & 0
\end{array}\right], \mathbf{D}^{0}=\left[\begin{array}{cccccccc}
1 & 0 & 0 & 0 & 0 & 0 & \cdots & 0 \\
0 & 1 & 0 & 0 & 0 & 0 & \cdots & 0 \\
0 & 0 & 1 & 0 & 0 & 0 & \cdots & 0 \\
0 & 0 & 0 & 1 & 0 & 0 & \cdots & 0 \\
0 & 0 & 0 & 0 & 1 & 0 & \cdots & 0 \\
0 & 0 & 0 & 0 & 0 & 1 & \cdots & 0 \\
\vdots & \vdots & \vdots & \vdots & \vdots & \ddots & \ddots & 0 \\
0 & 0 & 0 & 0 & 0 & 0 & \cdots & 1
\end{array}\right], \\
& \mathbf{T}=\left[\begin{array}{c}
\mathbf{T}\left(x_{0}\right) \\
\mathbf{T}\left(x_{1}\right) \\
\vdots \\
\mathbf{T}\left(x_{N}\right)
\end{array}\right]=\left[\begin{array}{cccc}
1 & x_{0} & \ldots & x_{0}^{N} \\
1 & x_{1} & \ldots & x_{1}^{N} \\
1 & \vdots & \ldots & \vdots \\
1 & x_{N} & \ldots & x_{N}^{N}
\end{array}\right], \mathbf{D}_{(\gamma)}=\left[\begin{array}{ccccc}
0 & 0 & 0 & \ldots & 0 \\
0 & \frac{\Gamma(2)}{\Gamma(2-\gamma)} & 0 & \ldots & 0 \\
0 & 0 & \frac{\Gamma(3)}{\Gamma(3-\gamma)} & \ldots & 0 \\
\vdots & \vdots & \vdots & \ddots & \vdots \\
0 & 0 & 0 & \cdots & \frac{\Gamma(N)}{\Gamma(N-\gamma)}
\end{array}\right] .
\end{aligned}
$$

By using equation (18), we obtain the following relation:

$$
\mathbf{Y}^{(k)}(x)=\mathbf{T}(x) \mathbf{D}^{k} \mathbf{M C} .
$$

By substituting the Bernoulli collocation points given by

$$
x_{i}=a+\frac{(b-a) i}{N}, \quad i=0,1, \ldots, N
$$

into equation (21), we obtain

$$
\mathbf{Y}^{(k)}\left(x_{i}\right)=\mathbf{T}\left(x_{i}\right) \mathbf{D}^{k} \mathbf{M C}, \quad k=0, \gamma, 2 .
$$

and the compact form of the relation (23) becomes

$$
\mathbf{Y}^{(k)}=\mathbf{T D}^{k} \mathbf{M C}, \quad k=0, \gamma, 2 .
$$

In this way, the unknown Bernoulli coefficients $c_{n}$, $n=1,2, \ldots, N+1$ are obtained by solving the system. Then, these coefficients are substituted into (14), and the approximate solution is obtained. For more details, see [36].

\section{Simulation Results and Discussion}

Bernoulli collocation method for obtaining the solution of fractional viscoelastic beam equation is employed. Hence, displacement analysis of a forced fractional viscoelastic beam is investigated by taking into account the different moving force loads, different values of internal damping coefficient, and different values of fractional order of derivative. Obtained results are simulated and presented in the tables and graphical forms. The velocity, from left to right, of the external moving force on beam $v(t)$ is considered as $\sin (\pi t)$. In order to observe the dynamic response of the viscoelastic beams under the different intensity of external moving force, the intensity constant of the external moving force load on the beam is involved to computation as $P=1,25,50$. Also, the values in Tables $1-6$ are computed on $x=0.5$, which is the middle point of the fractional viscoelastic beams. Observed duration of time is $t_{f}=1$. In the first case, forced displacement analysis of a polybutadiene beam is observed for different values of moving force load and results are presented in Table 1. The length and material density of the fractional viscoelastic beam are taken into account as $\ell=1 \mathrm{~m}$ and $\rho=160 \mathrm{~kg} / \mathrm{m}^{3}$, respectively. The cross-sectional area $A$ is $0.72 \mathrm{~m}^{2}$, moment of inertia $J$ is $(0.1)^{4} / 12$, and Young's modulus $E$ is $8.15 \times 10^{5}$ for a fractional viscoelastic polybutadiene beam. Also, the order of fractional derivative $\gamma$ is evaluated as 0.528 for the results in Figure 2 and Tables 1 and 3. By observing Figure 2, it is concluded that while the intensity of the external moving load force increases, namely, $P$ is 1 to 25 and 50, the displacement of the fractional viscoelastic polybutadiene beam also increases. Also, parallel observation results to Figure 2 are obtained by taking into account Table 1 . For example, on the moment $t=0.5$, the amount of the displacement of the polybutadiene beam is measured as 0.0011 for $P=1,0.028$ for $P=25$, and 0.056 for $P=50$. This observation is valid the entire time interval $t=0, \ldots, 1$ for polybutadiene beam. Also, the effect of internal damping on the displacement is presented in Table 3 for polybutadiene beam. The internal damping 
TABLe 1: Some values of $w(t, x)$ for $P=1,25,50$ (for a polybutadiene beam).

\begin{tabular}{lccr}
\hline$t$ & $w_{P=1}$ & $w_{P=25}$ & $w_{P=50}$ \\
\hline 0.1 & 0.0000252 & 0.0006320 & 0.0012640 \\
0.2 & 0.0001761 & 0.0044025 & 0.0088050 \\
0.3 & 0.0004637 & 0.0115934 & 0.0231868 \\
0.4 & 0.0008058 & 0.0201452 & 0.0402904 \\
0.5 & 0.0011150 & 0.0278772 & 0.0557545 \\
0.6 & 0.0013509 & 0.0337745 & 0.0675490 \\
0.7 & 0.0015294 & 0.0382361 & 0.0764721 \\
0.8 & 0.0017094 & 0.0427356 & 0.0854712 \\
0.9 & 0.0019330 & 0.0483262 & 0.0966525 \\
1.0 & 0.0020263 & 0.0506574 & 0.1013150 \\
\hline
\end{tabular}

TABle 2: Some values of $w(t, x)$ for $P=1,25,50$ (for a butyl B252 beam).

\begin{tabular}{lccr}
\hline$t$ & $w_{P=1}$ & $w_{P=25}$ & $w_{P=50}$ \\
\hline 0.1 & 0.0000202 & 0.0005053 & 0.0010107 \\
0.2 & 0.0001407 & 0.0035177 & 0.0070354 \\
0.3 & 0.0003701 & 0.0092531 & 0.0185064 \\
0.4 & 0.0006421 & 0.0160530 & 0.0321061 \\
0.5 & 0.0008865 & 0.0221645 & 0.0443289 \\
0.6 & 0.0010709 & 0.0267724 & 0.0535448 \\
0.7 & 0.0012079 & 0.0301981 & 0.0603962 \\
0.8 & 0.0013450 & 0.0336249 & 0.0672498 \\
0.9 & 0.0015159 & 0.0378992 & 0.0757984 \\
1.0 & 0.0015817 & 0.0395447 & 0.0790893 \\
\hline
\end{tabular}

Table 3: Some values of $w(t, x)$ for different values of $\mu$ for $P=1$ (for a polybutadiene beam).

\begin{tabular}{lccccc}
\hline$t \mu$ & 0.2 & 0.4 & 0.6 & 0.8 & 1.0 \\
\hline 0.1 & 0.00002490 & 0.00002424 & 0.00002362 & 0.00002303 & 0.00002248 \\
0.2 & 0.00017050 & 0.00016104 & 0.00015242 & 0.00014454 & 0.00013732 \\
0.3 & 0.00044042 & 0.00040252 & 0.00036954 & 0.00034070 & 0.00031537 \\
0.4 & 0.00074872 & 0.00065975 & 0.00058620 & 0.00052491 & 0.00047341 \\
0.5 & 0.00101132 & 0.00085673 & 0.00073583 & 0.00064002 & 0.00056311 \\
0.6 & 0.00119499 & 0.00097352 & 0.00081005 & 0.00068700 & 0.00059256 \\
0.7 & 0.00132326 & 0.00104456 & 0.00085035 & 0.00071106 & 0.00060830 \\
0.8 & 0.00145932 & 0.00113646 & 0.00092248 & 0.00077452 & 0.00066797 \\
0.9 & 0.00164541 & 0.00128775 & 0.00105863 & 0.00090275 & 0.00079071 \\
1.0 & 0.00171362 & 0.00134071 & 0.00111291 & 0.00096462 & 0.00086285 \\
\hline
\end{tabular}

TABle 4: Some values of $w(t, x)$ for different values of $\mu$ for $P=1$ (for a butyl B252 beam).

\begin{tabular}{|c|c|c|c|c|c|}
\hline$t \mu$ & 0.2 & 0.4 & 0.6 & 0.8 & 1.0 \\
\hline 0.1 & 0.00001990 & 0.00001935 & 0.00001885 & 0.00001837 & 0.00001791 \\
\hline 0.2 & 0.00013609 & 0.00012833 & 0.00012126 & 0.00011482 & 0.00010894 \\
\hline 0.3 & 0.00035096 & 0.00031992 & 0.00029302 & 0.00026958 & 0.00024907 \\
\hline 0.4 & 0.00059532 & 0.00052271 & 0.00046302 & 0.00041352 & 0.00037212 \\
\hline 0.5 & 0.00080177 & 0.00067617 & 0.00057862 & 0.00050179 & 0.00044045 \\
\hline 0.6 & 0.00094391 & 0.00076493 & 0.00063394 & 0.00053604 & 0.00046136 \\
\hline 0.7 & 0.00104091 & 0.00081711 & 0.00066268 & 0.00055279 & 0.00047222 \\
\hline 0.8 & 0.00145932 & 0.00088619 & 0.00071738 & 0.00060155 & 0.00066797 \\
\hline 0.9 & 0.00128591 & 0.00100283 & 0.00082334 & 0.00070197 & 0.00051857 \\
\hline 1.0 & 0.00133374 & 0.00104133 & 0.00086475 & 0.00075070 & 0.00061500 \\
\hline
\end{tabular}

coefficient is evaluated from 0.2 to 1 and by examining Table 3; it reveals that when internal damping coefficient increases, the displacement of the polybutadiene beam decreases under the same conditions. The effect of the fractional order to system is observed from Table 5 and it can be concluded that while increasing the values of the 
TABLe 5: Some values of $w(t, x)$ for different values of $\gamma$ for $P=1$ (for a polybutadiene beam).

\begin{tabular}{lccccc}
\hline$t \gamma$ & 0.2 & 0.4 & 0.6 & 0.8 & 1.0 \\
\hline 0.1 & 0.00002528 & 0.00002528 & 0.00002528 & 0.00002527 & 0.00002527 \\
0.2 & 0.00017611 & 0.00017610 & 0.00017609 & 0.00017607 & 0.00017604 \\
0.3 & 0.00046379 & 0.00046376 & 0.00046371 & 0.00046363 & 0.00046349 \\
0.4 & 0.00080596 & 0.00080588 & 0.00080575 & 0.00080556 & 0.00080526 \\
0.5 & 0.00111539 & 0.00111523 & 0.00111499 & 0.00135034 & 0.00111416 \\
0.6 & 0.00135146 & 0.00135120 & 0.00135084 & 0.00152864 & 0.00134969 \\
0.7 & 0.00153010 & 0.00152973 & 0.00152925 & 0.00170852 & 0.00152788 \\
0.8 & 0.00171024 & 0.00170978 & 0.00170920 & 0.00193209 & 0.00079071 \\
0.9 & 0.00193399 & 0.00193345 & 0.00193281 & 0.00202538 & 0.00193132 \\
1.0 & 0.00202731 & 0.00202671 & 0.00202606 & \\
\hline
\end{tabular}

TABLe 6: Some values of $w(t, x)$ for different values of $\gamma$ for $P=1$ (for a butyl B252 beam).

\begin{tabular}{lccccc}
\hline$t \gamma$ & 0.2 & 0.4 & 0.6 & 0.8 & 1.0 \\
\hline 0.1 & 0.00002021 & 0.00002021 & 0.00002021 & 0.00002021 & 0.00002021 \\
0.2 & 0.00014071 & 0.00014071 & 0.00014070 & 0.00014069 & 0.00014066 \\
0.3 & 0.00037016 & 0.00037014 & 0.00037011 & 0.00037006 & 0.00036997 \\
0.4 & 0.00064221 & 0.00064216 & 0.00064208 & 0.00064196 & 0.00064177 \\
0.5 & 0.00088676 & 0.00088666 & 0.00088651 & 0.00088629 & 0.00088598 \\
0.6 & 0.00107119 & 0.00107102 & 0.00107079 & 0.00120741 & 0.00107007 \\
0.7 & 0.00120833 & 0.00120809 & 0.00120779 & 0.00134441 & 0.00120693 \\
0.8 & 0.00134549 & 0.00134520 & 0.00134484 & 0.00151536 & 0.00134392 \\
0.9 & 0.00151653 & 0.00151620 & 0.00151580 & 0.00158121 & 0.00151489 \\
1.0 & 0.00158239 & 0.00158202 & 0.00158162 & 0.00158082 \\
\hline
\end{tabular}

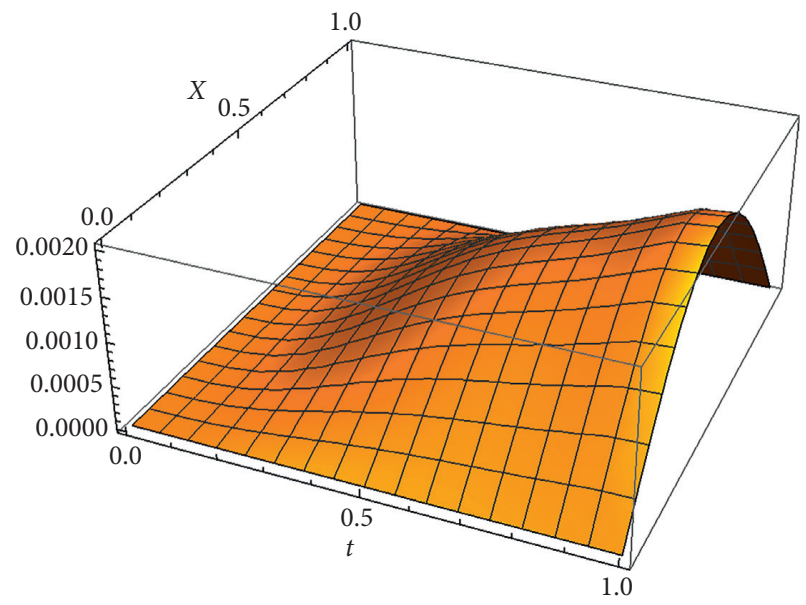

(a)

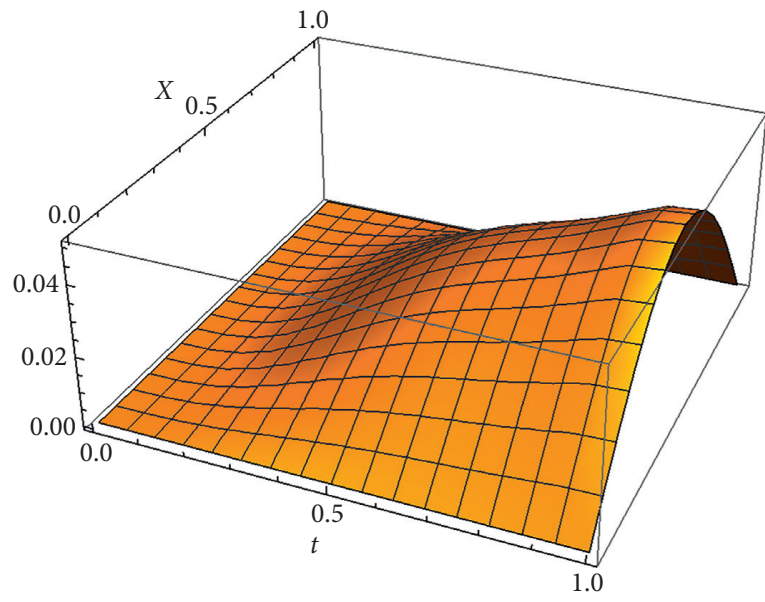

(b)

Figure 2: Continued. 


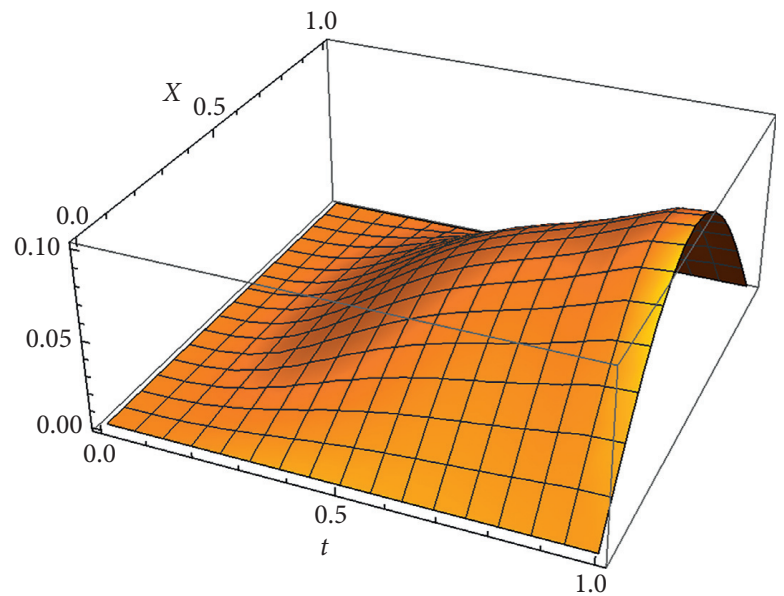

(c)

Figure 2: Displacements of a polybutadiene beam for $P=1,25,50$.

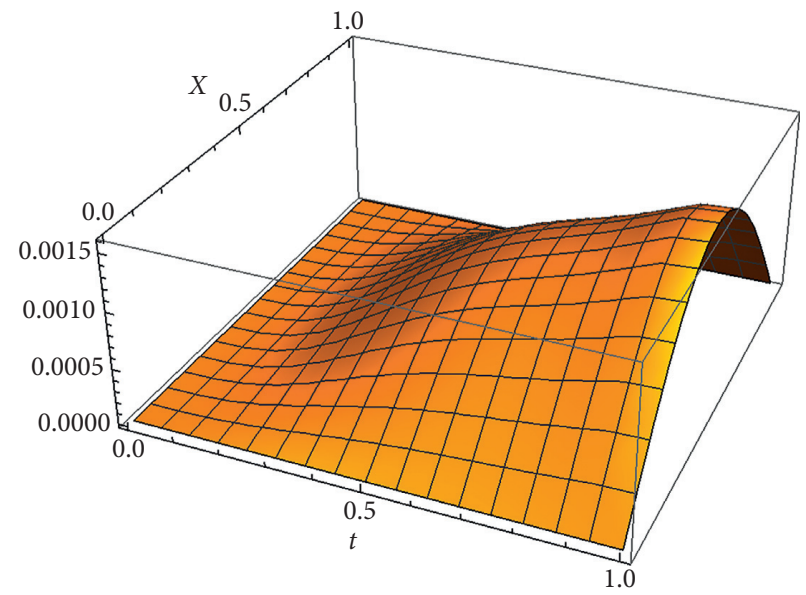

(a)

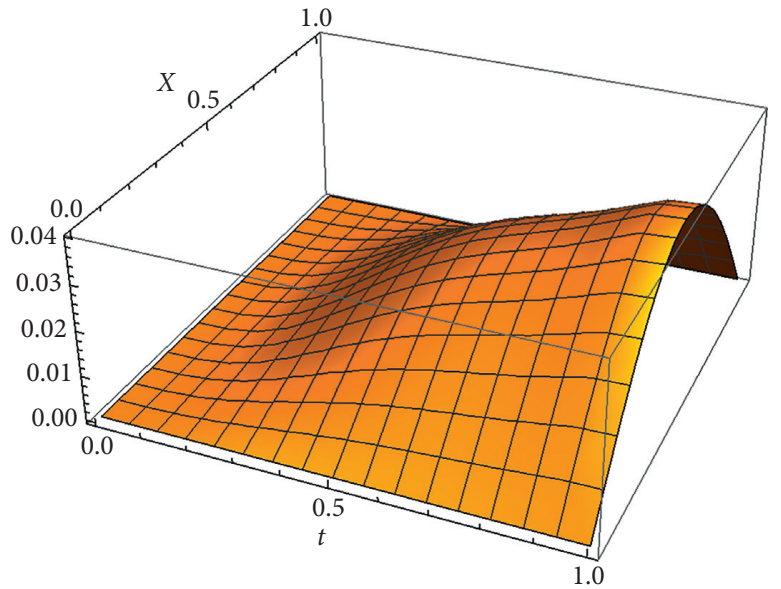

(b)

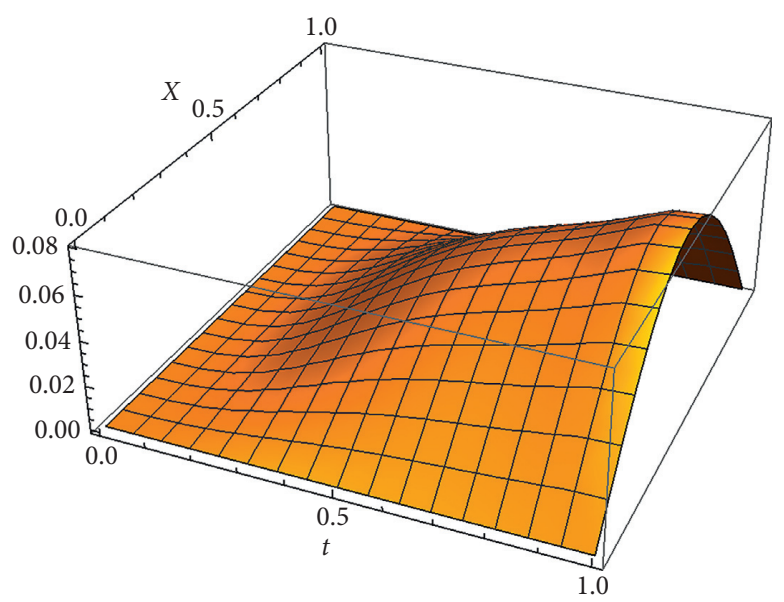

(c)

Figure 3: Displacements of a butyl B252 beam for $P=1,25,50$. 
fractional derivative, the value of the displacement is decreasing. In the second case, a butyl B252 beam is taken into account by the coefficients; the cross-sectional area $A$ is $0.72 \mathrm{~m}^{2}$, moment of inertia $J$ is $(0.1)^{4} / 12$, and Young's modulus $E$ is $1.05 \times 10^{6}$. The order of fractional derivative $\gamma$ is considered as 0.519 for Figure 3 and Tables 2 and 4 . By checking Figure 3, it is easy to see that displacements corresponding to much bigger intensity of moving force load are much bigger. For example, on the moment $t=0.5$, while $P=1$ to $P=25,50$, corresponding displacements are calculated as $0.00089,0.022$, and 0.044 , respectively. This is effective along the observation duration. In Table 4, some results related to the effect of internal damping are presented and internal damping coefficient is included in the computation as 0.2 to 1 . After looking at Table 4 , it is concluded that while internal damping coefficient decreases, the displacement of the butyl B252 beam increases and relation between the effects of internal damping and displacements is inversely proportional. The relation between the displacement and fractional order in the system is vice versa. As understood from Table 6, while decreasing the values of the fractional derivative, the value of the displacement is increasing. These observation results of the present study are also compatible with the results existing in the literature. By taking into account Tables 1-6 and Figures 2 and 3 and comparing these two kinds of fractional viscoelastic beams, it is seen that the polybutadiene beam has more greater displacements than butyl B252 beam under same conditions. Also, the effect of internal damping coefficient is more visible on the butyl B252 beam according to polybutadiene beam. These observations make clear that butyl B252 beam is stronger and preferable than the polybutadiene beam.

\section{Conclusion}

In this study, the Bernoulli collocation method as a new solution method for obtaining the approximate solution of a fractional viscoelastic beam model subjected to moving force load is employed. Dynamic response analysis of the fractional viscoelastic beam model is investigated for two different specific beams: polybutadiene beam and butyl B252 beam. Displacement analysis of a point on the fractional viscoelastic beams is studied for different moving force loads and also effect of the internal damping to displacement is observed for different internal damping coefficients. Moreover, dynamic response of the fractional viscoelastic beam is examined for different values of the fractional order. Obtained results are presented in tables and graphics and results reveal that Bernoulli collocation method is very effective and powerful solution method for obtaining the solution of fractional order viscoelastic beam models. After observing Figures 2 and 3, it is easy to conclude that as the moving force load increases, the displacement of a point on the beams also increases. Also, numerical results, presented in Tables 1-4, show that under the same moving force load with the same internal damping effect, the displacement of a point on the polybutadiene beam is greater than that corresponding to butyl B252 beam. Moreover, under the same moving force load, changes in the displacements of a point on the beams are examined in the aspect of different internal damping effects and observations made clear that butyl B252 beam better reflects the effect of internal damping to displacement of a point on the beam. By comparing polybutadiene beam and butyl B252 beam, it is concluded that polybutadiene beam is more open to destructive effects of vibrations under the same conditions with the butyl B252 beam.

\section{Data Availability}

The data used to support the findings of this study are included within the article.

\section{Conflicts of Interest}

The authors declare that they have no conflicts of interest.

\section{Authors' Contributions}

The authors completed this study and wrote and approved the final version of the manuscript.

\section{References}

[1] O. A. Bauchau and J. I. Craig, Structural Analysis, SpringerVerlag, New York, NY, USA, 2009.

[2] J. Lubliner and P. Papadopoulos, Introduction to Solid Mechanics:An Integrated Approach, Springer-Verlag, New York, NY, USA, 2014.

[3] R. Rowland Jr., Principal of Solid Mechanics, CRC Press, Boca Raton, FL, USA, 2000.

[4] T. Blaszczyk, J. Siedlecki, and H. Sun, "An exact solution of fractional Euler-Bernoulli equation for a beam with fixedsupported and fixed-free ends," Applied Mathematics and Computation, vol. 396, p. 125932, 2021.

[5] G. Radenkovic and A. Borkovic, "On the analytical approach to the linear analysis of an arbitrarily curved spatial Bernoulli Euler beam," Applied Mathematical Modelling, vol. 77, pp. 1603-1624, 2020.

[6] E. Pan and P. R. Heyliger, "Free vibrations of simply supported and multilayered magneto-electro-elastic plates," Journal of Sound and Vibration, vol. 252, no. 3, pp. 429-442, 2002.

[7] A. R. Annigeri, N. Ganesan, and S. Swarnamani, "Free vibration behaviour of multiphase and layered magneto-electro-elastic beam," Journal of Sound and Vibration, vol. 299, no. 1, pp. 44-63, 2007.

[8] G. W. Griffiths and W. E. Schiesser, Traveling Wave Analysis of Partial Differential Equations, Academic Press, Cambridge, MA, USA, 2011.

[9] C.-G. Zhang, "Boundary feedback stabilization of the undamped Timoshenko beam with both ends free," Journal of Mathematical Analysis and Applications, vol. 326, no. 1, pp. 488-499, 2007.

[10] A. Capsoni, G. Maria Viganò, and K. Bani-Hani, "On damping effects in Timoshenko beams," International Journal of Mechanical Sciences, vol. 73, pp. 27-39, 2013.

[11] K. Morfidis, "Vibration of Timoshenko beams on three-parameter elastic foundation," Computers \& Structures, vol. 88, no. 5-6, pp. 294-308, 2010. 
[12] M. Ishaquddin and S. Gopalakrishnan, "A novel weak form quadrature element for gradient elastic beam theories," Applied Mathematical Modelling, vol. 77, pp. 1-16, 2020.

[13] B. Martin and A. Salehian, "Techniques for approximating a spatially varying Euler-Bernoulli model with a constant coefficient model," Applied Mathematical Modelling, vol. 79, pp. 260-283, 2020.

[14] J. Gahleitner and J. Schoeftne, "An anisotropic beam theory based on the extension of Boleyr's method," Composite Structures, vol. 243, pp. 112-149, 2020.

[15] B. Wang, X. Luo, Y. Liu, and Z. Yang, "Thickness-variable composite beams for vibration energy harvesting," Composite Structures, vol. 244, p. 112232, 2020.

[16] A. Gemant, "XLV.On fractional differentials," The London, Edinburgh, and Dublin Philosophical Magazine and Journal of Science, vol. 25, no. 168, pp. 540-549, 1938.

[17] C. Yu, J. Zhang, Y. Chen, Y. Feng, and A. Yang, "A numerical method for solving fractional-order viscoelastic Euler Bernoulli beams," Chaos, Solitons \& Fractals, vol. 128, pp. 275279, 2016.

[18] R. K. Praharaj and N. Datta, "Dynamic response of plates resting on a fractional viscoelastic foundation and subjected to a moving load," Mechanics Based Design of Structures and Machines, pp. 1-16, 2020.

[19] R. K. Praharaj and N. Datta, "Dynamic response spectra of fractionally damped viscoelastic beams subjected to moving load," Mechanics Based Design of Structures and Machines, vol. 2020, Article ID 1725563, 15 pages, 2020.

[20] J. Xu and J. Li, "Stochastic dynamic response and reliability assessment of controlled structures with fractional derivative model of viscoelastic dampers," Mechanical Systems and Signal Processing, vol. 72-73, pp. 865-896, 2016.

[21] M. Ajri, M. M. Fakhrabadi, and A. Rastgoo, "Analytical solution for nonlinear dynamic behavior of viscoelastic nanoplates modeled by consistent couple stress theory," Latin American Journal of Solids and Structures, vol. 15, pp. 1-23, 2018.

[22] C. Chazal and R. M. Pitti, "Integral approach for time dependent materials using finite element method," Journal of Theorical and Applied Mechanics, vol. 49, pp. 1029-1048, 2011.

[23] Y. Lei, M. I. Friswell, and S. Adhikari, "A Galerkin method for distributed systems with non-local damping," International Journal of Solids and Structures, vol. 43, no. 11-12, pp. 3381-3400, 2006.

[24] O. Martin, "A modified variational iteration method for the analysis of viscoelastic beams," Applied Mathematical Modelling, vol. 40, no. 17-18, pp. 7988-7995, 2016.

[25] D. D. Demir, N. Bildik, and B. G. Sýnýr, "Linear dynamical analysis of fractionally damped beams and rods," Journal of Engineering Mathematics, vol. 85, no. 1, p. 13147, 2014.

[26] S. Mareishi, M. Rafiee, X. Q. He, and K. M. Liew, "Nonlinear free vibration, postbuckling and nonlinear static deflection of piezoelectric fiber-reinforced laminated com- posite beams," Composites Part B: Engineering, vol. 59, p. 12332, 2014.

[27] J. K. Freundlich, "Dynamic response of a simply supported viscoelastic beam of a fractional derivative type to a moving force load," Journal of Theoretical and Applied Mechanics, vol. 54, no. 4, pp. 1433-1445, 2016.

[28] Z.-F. Liang and X.-Y. Tang, "Analytical solution of fractionally damped beam by Adomian decomposition method," Applied Mathematics and Mechanics, vol. 28, no. 2, pp. 219-228, 2007.

[29] M. A. Foda and Z. Abduljabbar, "A dynamic green function formulation for the response of a beam structure to a moving mass," Journal of Sound and Vibration, vol. 210, no. 3, pp. 295-306, 1998.

[30] J. Freundlich, "Transient vibrations of a fractional KelvinVoigt viscoelastic cantilever beam with a tip mass and subjected to a base excitation," Journal of Sound and Vibration, vol. 438, pp. 99-115, 2019.

[31] W. Sumelka, T. Blaszczyk, and C. Liebold, "Fractional EulerBernoulli beams: theory, numerical study and experimental validation," European Journal of Mechanics-A: Solids, vol. 54, pp. 243-251, 2015.

[32] M. Sameeh and A. Elsaid, "Chebyshev collocation method for parabolic partial integrodifferential equations," Advances in Mathematical Physics, vol. 2016, Article ID 7854806, 7 pages, 2016.

[33] F. Toutounian, E. Tohidi, and S. Shateyi, "A collocation method based on the Bernoulli operational matrix for solving high-order linear complex differential equations in a rectangular domain," Abstract and Applied Analysis, vol. 2013, Article ID 823098, 12 pages, 2013.

[34] Ö. Oruç, A. Esen, and F. Bulut, "Numerical investigation of dynamic Euler-Bernoulli equation via 3-Scale Haar wavelet collocation method," Hacettepe Journal of Mathematics and Statistics, vol. 50, no. 1, pp. 1-21, 2021.

[35] R. L. Bagley and P. J. Torvik, "A theoretical basis for the application of fractional calculus to viscoelasticity," Journal of Rheology, vol. 27, no. 3, pp. 201-210, 1983.

[36] B. Zogheib, E. Tohidi, and S. Shateyi, "Bernoulli collocation method for solving linear multidimensional diffusion and wave equations with dirichlet boundary conditions," Advances in Mathematical Physics, vol. 2017, Article ID 5691452, 15 pages, 2017. 